\title{
Negative differential conductivity in Fermi liquid in the presence of magnetic field
}

\author{
N. N. Konobeeva ${ }^{1}$, M. B. Belonenko ${ }^{1,2}$ \\ ${ }^{1}$ Volgograd State University, Volgograd, Russia \\ ${ }^{2}$ Volgograd Institute of Business, Volgograd, Russia \\ yana_nn@inbox.ru, mbelonenko@yandex.ru
}

PACS 71.10.Ay, 72.20.-i, 73.40.Gk

DOI 10.17586/2220-8054-2016-7-3-422-426

\begin{abstract}
In this paper we, study the response of a Fermi liquid under the influence of an external magnetic field applied to the external electric field. The dispersion law of the Fermi liquid is obtained via AdS/CFT correspondence. The regions of the negative differential conductivity on the current-voltage characteristic were observed. The possibility of terahertz pulse generation in such systems was shown for a wide range of magnetic field strengths.
\end{abstract}

Keywords: Fermi liquid, AdS/CFT correspondence, differential negative conductivity.

Received: 15 January 2016

Revised: 22 January 2016

\section{Introduction}

The impetus for writing this paper was the fact that we can control the important characteristics of an electron subsystem (including conductivity) by controlling the spectrum of charge carriers, for example with a magnetic field. From a physical point of view, the reason is a phenomenon similar to the Hall Effect, namely the deflection of electrons under the influence of an electromagnetic field pulse. It should be noted that in the last few years there has been increased interest in this area [1].

One such characteristic is the current-voltage characteristic for a well-established contact with considerable substance. In this case, as will be shown below, the current-voltage characteristic is determined by the imaginary part of the Green's function.

At the same time, great progress in the Green's functions calculation for different systems - with many transformation of the corresponding fields when the coordinates change (scalar, spinor, etc.) - was made by the AdS/CFT correspondence between the conformal field theory and the theory of superstrings [2,3]. This fact allows experimental verification for some of the associated consequences using methods of solid state physics. Usually, the research in this area is focused on the conductivity study [4]. However, studies on other characteristics, which can be subjected to experimental tests, are still lacking.

Based on the above-mentioned facts, the purpose of this paper is to study the response of a Fermi liquid under the influence of an external magnetic field applied to the external electric field in circumstances where the amplitude of the external alternating fields are small and linear response theory is not applicable. The dispersion law is obtained via AdS/CFT correspondence [2,3,5].

\section{Basic equation}

In order to study tunneling effects, the Hamiltonian of our model has the following form:

$$
H=\sum_{p} E_{p}^{A} a_{p}^{+} a_{p}+\sum_{q} E_{q}^{B} b_{q}^{+} b_{q}+\sum_{p q} T_{p q}\left(a_{p}^{+} b_{q}+b_{q}^{+} a_{q}\right),
$$

where $a_{p}^{+} ; a_{p}$ are the creation and annihilation operators with momentum $p$ in the metal; $E_{p}^{A}$ is the electron spectrum of a metal; $T_{p q}$ is the matrix element of the tunneling operator between the states $p$ and $q ; b_{q}^{+} ; b_{q}$ are the creation and annihilation operators with momentum $q$ of a substance in contact with the metal; $E_{p}^{B}$ is the electron spectrum of the substance.

Following the concepts laid out in [6], we find that the current is [7]:

$$
J=4 \pi e|T|^{2} \sum_{p q} \int_{-\infty}^{\infty} d E \operatorname{Im} G^{A}(p, E+e V) \cdot \operatorname{Im} G^{B}(q, E)\left(n_{f}(E)-n_{f}(E+e V),\right.
$$


where $G^{A}$ is the Green's function for a superlattice, $G^{B}$ is the Green's function for a Fermi liquid, $V$ is the voltage applied to a test substance, is the electron charge.

The dispersion law for a superlattice has the following form [8]:

$$
E_{p}^{A}=\varepsilon_{0}-\Delta \cos (p)
$$

where $\varepsilon_{0}$ is the energy of quantum well electrons, $\Delta$ is the tunneling integral determined by the overlap of the electron wave functions of neighboring wells, momentum is directed along $z$-axis.

As a result, we obtain the following expression for tunneling current:

$$
J=4 \pi e|T|^{2} \sum_{p q} \int_{-\infty}^{\infty} d E \frac{1}{\sqrt{1-\left(\frac{\varepsilon_{0}-E-e V}{\Delta}\right)}} \cdot \operatorname{Im} G^{B}(q, E)\left(n_{f}(E)-n_{f}(E+e V)\right) .
$$

So, after the calculation of the integral in Eq. (4), it is easy to obtain the current-voltage characteristic of the superlattice and Fermi liquid contact.

\section{Excitation spectrum from the AdS/CFT correspondence}

The Green's function of the systems near the quantum critical point for example "strange metals" can be found in the framework of the holographic approach. High temperature superconductors in the quantum critical region, and other similar materials, can be dual with models of superstring theory with gravity $[9,10]$. Duality is understood here in the sense of the gravity theory with matter (fermions, scalar fields) in space-time with a certain dimension being equivalent (in terms of mean values) to some field theory on the boundary of space-time. This gives one the opportunity to obtain an expression for the average values of the dual variables (understood in the usual sense of the conformal field theory) at the boundary and, therefore, the Green's function [11,12] can be calculated for the wave functions of the perturbation field in the bulk material.

The action for the vector field can be written as:

$$
S=\frac{1}{2 k^{2}} \int d^{4} x \sqrt{-g}\left(R+\frac{6}{R^{2}}-\frac{R^{2}}{g_{f}^{2}} F_{M N} F^{M N}\right),
$$

here $g_{F}^{2}$ is the effective dimensionless calibration constant, $R$ is the curvature radius of $\operatorname{AdS}_{4}$.

The motion equations following from Eq. (5) can be solved by geometry, corresponding to a dyonic black hole with electric and magnetic charge:

$$
d s^{2}=g_{M N} d x^{M} d x^{N}=\frac{r^{2}}{R^{2}}\left(-f d t^{2}+d x^{2}+d y^{2}\right)+\frac{R^{2}}{r^{2}} \frac{d r^{2}}{f}
$$

where $f$ is the redshift coefficient, and:

$$
\begin{gathered}
f=1+\frac{Q^{2}+H^{2}}{r^{4}}-\frac{M}{r^{3}}, \quad A_{t}=\mu\left(1-\frac{r_{0}}{r}\right), \quad A_{y}=h x, \quad A_{x}=A_{r}=0, \\
M=r_{0}^{3}+\frac{Q^{2}+H^{2}}{r_{0}},
\end{gathered}
$$

where $Q, H$ are the electric and magnetic charge of the black hole, $\mu$ is the black hole chemical potential, $h$ is the magnetic field of the black hole, $r_{0}$ is the horizon radius.

The Green's function can be obtained from the Dirac equation, and near the Fermi surface, is:

$$
G(\omega)=\frac{-h_{1} h_{2} \nu_{F}}{\omega-\nu_{F} k_{\perp}-h_{2} \nu_{F} e^{i \theta-i \pi \nu} \omega^{2 \nu}}
$$

where $v_{F}$ is the Fermi velocity, $k_{\perp}=k-k_{F}, k_{F}$ is the Fermi momentum, $k$ is the momentum module, $\nu$ is the conformal dimension, $\theta$ and $h_{1,2}$ are the positive constant, $\omega$ is the frequency.

The effect of the magnetic field is determined by the discretization of the momentum:

$$
k \rightarrow k_{e f f}=\sqrt{2|q h| l}, \quad l \in \mathbb{N},
$$

$l$ is the Landau level index. These discrete values of $k$ are the analog of the well-known Landau levels, which occur in magnetic systems.

Following the concepts which were delineated in $[2,13]$, we find that the imaginary part of the Green's function is: 


$$
\operatorname{Im} G(\omega)=\frac{-h_{1} h_{2} \nu_{F}^{2} \omega^{2 \nu} \sin (\theta-\pi \nu)}{\left(\omega-\nu_{F} k_{\perp}-h_{2} \nu_{F} \omega^{2 \nu} \cos (\theta-\pi \nu)\right)^{2}+h_{2}^{2} \nu_{F}^{2} \omega^{4 \nu} \sin ^{2}(\theta-\pi \nu)} .
$$

\section{The results of numerical analysis and discussion}

The current-voltage characteristics of the contact between the superlattice and a Fermi liquid for different tunneling integral $\Delta$ values are shown in Fig. 1.

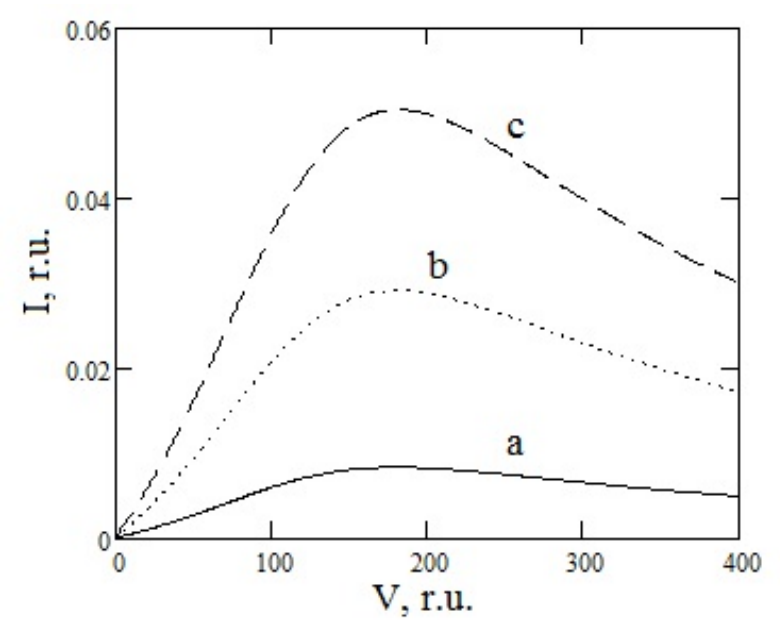

FIG. 1. Current-voltage characteristics of the tunneling contact for $h=1.5$ r.u., $\nu=1.3$ :

a) $\Delta=0.01$ r.u.; b) $\Delta=0.03$ r.u.; c) $\Delta=0.05$ r.u.

A decreasing region in the CVC can be seen, which points to differential negative conductivity, which is specific for all substances having a periodical dispersion law. Behavior of the curve is determined by the tunneling integral $\Delta-$ the higher the value, the greater the current.

CVC dependence on the magnetic field is presented in Fig. 2.



FIG. 2. Current-voltage characteristics of the tunneling contact for $\Delta=0.05$ r.u., $\nu=1.3$ :

a) $h=1.0$ r.u.; b) $h=1.5$ r.u.; c) $h=2.0$ r.u.

According to the observed dependence, it can be concluded that with a stronger magnetic field, the tunneling current also increases, and hence, differential resistance decreases.

The dependence of the tunneling current on the applied voltage for different conformal dimension $\nu$ values is given in Fig. 3.

As can be seen from the graphs, for some values of parameter $\nu$, there appear two regions with differential negative conductivity, and in the case $\nu=1.1$, there is even a region with an absolute negative conductivity (ANC). 


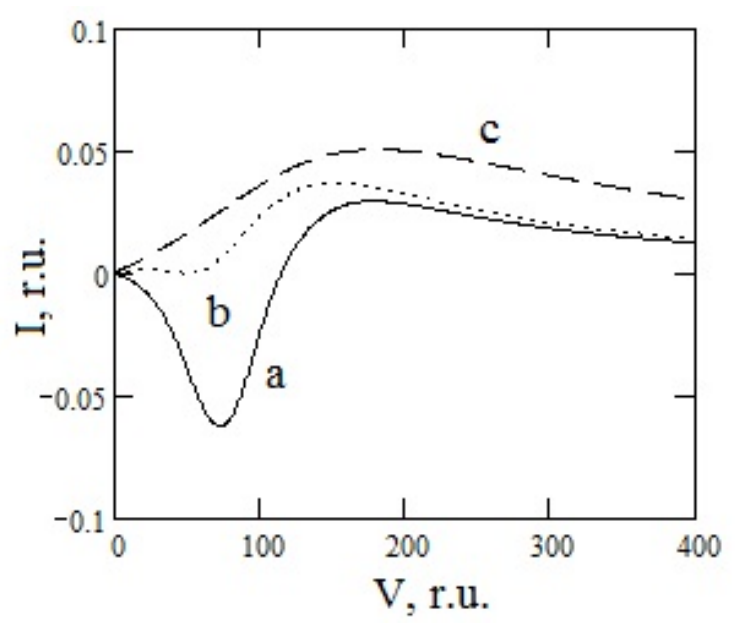

FIG. 3. Current-voltage characteristics of the tunneling contact for $\Delta=0.05$ r.u., $h=1.5$ r.u.:

a) $\nu=1.1$; b) $\nu=1.2$; c) $\nu=1.3$.

As noted in $[14,15]$, the uniform state system with an absolute negative conductivity is unstable due to the fact that the current-voltage characteristics with ANC has a region with negative differential conductivity of about zero field $E=0$. This instability usually leads to the formation of the electric field domains and to the stratification of the current lines [16]. This circumstance, according to [17,18], allows us to assume that the state with zero resistance and zero conductivity are spatially inhomogeneous, and arise from the development of instability in the initial homogeneous state with ANC. Thus, due to the value of the conformal dimension, we can control the negative differential resistance, and also the CVC's form, which significantly affects the contact properties.

Additionally, we can verify the condition for the existence of terahertz signals in our system. This condition, according to [19], can be written in the following form:

$$
R_{N}+R_{C}+R_{A}<0
$$

where $R_{N}$ is the negative differential resistance of the superlattice and Fermi liquid contact; $R_{C}$ is the contact resistance; $R_{A}$ is the radiation resistance of the antenna. According to [19], we choose $R_{C}=12.9 \mathrm{k} \Omega, R_{A}=80 \Omega$. Then, in order to satisfy the condition, we have:

$$
\begin{gathered}
R_{k p}=R_{C}+R_{A}=12.98 k \Omega, \\
\left|R_{C}\right|>R_{k p} .
\end{gathered}
$$

For all examined values of the magnetic field, this condition is satisfied; the greater the magnetic field, the smaller the negative differential resistance.

\section{Conclusion}

Here, we summarize our main results:

1. A method for calculating the Fermi liquid and superlattice contact CVC in the case of magnetic fields is presented.

2. In a Fermi liquid in the presence of constant magnetic field, there are regions of differential negative conductivity and absolute negative conductivity.

3. The conductivity of such contact strongly depends on the frequency of the magnetic field and also depends on the conformal dimension.

Terahertz pulses can be generated in this system for a wide range of voltage $V$. These pulses may have utility in a number of practical applications.

\section{Acknowledgements}

This work was supported by the Russian Foundation for Basic Research under project No. 16-32-00230 мол_a and project No. 16-07-01265. 


\section{References}

[1] Izyumov Yu.A., Letfulov B.M., Shipitsyn E.V., Chao K.A. A theory of ferromagnetism in the Hubbard model with infinite Coulomb interaction. Int. J. Mod. Phys., 1992, 6, P. 3479-3514.

[2] Policastro G., Son D.T., Starinets A.O. From AdS/CFT correspondence to hydrodynamics. J. High Energy Phys., 2002,9 , P. 43.

[3] Pal Sh.S. Model building in AdS/CMT: DC conductivity and Hall angle. Phys. Rev. D, 2011, 84, P. 126009.

[4] Sachdev S. What can gauge-gravity duality teach us about condensed matter physics. Annual Rev. Cond. Mat. Phys, 2012 , 3, P. 9.

[5] Nakayama Yu.A lecture note on scale invariance vs conformal invariance, 2013, http://arxiv.org/abs/1302.0884.

[6] Belonenko M.B., Konobeeva N.N., et al. Tunneling characteristics of a contact between a superlattice and non-Fermi liquid using the AdS/CFT correspondence. Mod. Phys. Let. B, 2014, 28, P. 1450170.

[7] Levitov L.S., Shitov A.V. Green's functions. Problems with solutions, Moscow: Fizmatlit, 2003, 392 p.

[8] Belonenko M.B., Mescheryakova N.E. Electromagnetic solitons in a system of quantum dots with taking into account the Hubbard interaction. J. Rus. Las. Res., 2008, 29, P. 544.

[9] Witten E. Anti-de Sitter space and holography. Adv. Theor. Math. Phys, 1998, 2, P. 253.

[10] Kulaxizi M., Parnachev A. Comments on Fermi Liquid from Holography. Phys. Rev. D, 2008, 78, P. 086004.

[11] Kulaxizi M., Parnachev A. Holographic Responses of Fermion Matter. Nucl. Phys. B, 2009, 815, P. 125.

[12] Charmousis C., Gouteraux B., et al. Effective Holographic, Theories for low-temperature condensed matter systems. J. High Energy Phys, 2010, 1011, P. 151.

[13] Hartnol S.A. Quantum critical dynamics from black holes, 2010, http://arxiv.org/abs/0909.3553v2.

[14] Gladun A.D., Ryzhij V.I. Absolute negative conductivity mechanism of the thin films in quantizing transverse field. JETP Lett., 1969, 57, P. 978.

[15] Ignatov .A., Romanov Yu.A. Absolute negative conductivity in semiconductor with superlattice. Radiophys. Quant. Electr., 1978, 21, P. 90.

[16] Volkov A.F., Kogan Sh.M. Physical phenomena in semiconductors with negative differential conductivity. UFN, 1968,96, P. 633.

[17] Andreev A.V., Aleiner I.L., Millis A.J. Dynamical symmetry breaking as the origin of the zero-dc-resistance state in an ac-driven system. Phys. Rev. Lett., 2003, 91, P. 056803.

[18] Bergeret F.S., Huckestein B., Volkov A.F. Current-voltage characteristics and the zero-resistance state in a two-dimensional electron gas. Phys. Rev. B, 2003, 67, P. 241303(R).

[19] Dragoman D., Dragoman M. Terahertz oscillations in semiconducting carbon nanotubes resonant-tunneling diodes. Physica E, 2004, 24, P. 282-289. 